\title{
Investigation on interlaminar shear strength properties of disc laser machined consolidated CF-PPS laminates
}

\author{
P. Jaeschke ${ }^{1 *}$, M. Kern ${ }^{1}$, U. Stute ${ }^{1}$, H. Haferkamp ${ }^{1}$, C. Peters ${ }^{2}$, A. S. Herrmann ${ }^{2}$ \\ ${ }^{1}$ Laser Zentrum Hannover e. V., Hollerithallee 8, 30419 Hannover, Germany \\ ${ }^{2}$ Faserinstitut Bremen e.V., Am Biologischen Garten 2, 28359 Bremen, Germany
}

Received 17 September 2010; accepted in revised form 8 December 2010

\begin{abstract}
In consequence of an increased interest in using endless carbon fibre reinforced thermoplastic composites (TPC), automated and highly productive processing technologies for cutting and trimming steps of consolidated materials are sought. In this paper, the influence on the thermal effect caused by laser cutting with respect to static strength properties of TPC based on a polyphenylene sulfide (PPS) matrix is studied. For the cutting experiments, consolidated TPC laminates at varying thicknesses up to $s=3.1 \mathrm{~mm}$ and a disc laser emitting at a wavelength of $\lambda=1030 \mathrm{~nm}$ at a maximum output power of $P_{\mathrm{L}}=2 \mathrm{~kW}$ are used. For the first time, the resulting magnitude of the heat affected zone (HAZ) at the cutting edge of the composite material is correlated with interlaminar shear strength tests. The results are compared to specimens prepared by milling and abrasive water jet cutting. Depending on the laminate thickness, the laser treated TPC samples show comparable properties to those of conventionally processed specimens. A reduced load bearing area, as a consequence of damaged fibre-matrix-adhesion due to laser impact, is identified as main factor for the reduction of interlaminar shear strengths for higher laminate thicknesses.
\end{abstract}

Keywords: polymer composites, processing technologies, mechanical properties, laser processing

\section{Introduction}

Endless carbon and glass fibre reinforced composite structures (CFRP, GFRP) are recognized as having the greatest lightweight construction potential of all materials. Hence, CFRP and GFRP are representing significant materials for all branches of the industry where large masses have to be moved or weight has to be saved. Besides the field of mobility, including the aircraft, the automotive, the marine and the railway sector, the need of components based on fibre reinforced composites is particularly increasing within the field of energy, e.g. wind turbines, heavy-duty pipeline elements for off-shore applications and electronics, as well as within the field of sports and leisure.

In particular CFRP is characterized by high stiffness and strength, excellent corrosion resistance as well as high static and dynamic loading. An outstanding property of CFRP can be found within the potential of significant weight reduction compared to metallic materials, e.g. up to $30 \%$ for aluminium. Thus, in order to contribute significantly to energy and $\mathrm{CO}_{2}$ savings by use of intelligent light weight constructions within the mobility sector as well as to supply optimized manufacturing processes, the use of CFRP will play an increasing role.

Although thermoset polymers mainly based on epoxy resins are representing the predominant kind of CFRP matrix materials, reinforced composites on the basis of thermoplastic polymers (TPC) are of rising interest for a quick and reliable component formability manufacture process. The potential of TPC parts inside the forming process is the ability of in-situ consolidation in just one process step.

\footnotetext{
${ }^{*}$ Corresponding author, e-mail: p.jaeschke@1zh.de
}

(c) BME-PT 
Thermoplastics offer several benefits such as uncritical and unlimited storage time, a better impact tolerance compared to thermoset matrices, a good ultimate strain performance, reduced crack propagation, excellent chemical resistance and quick forming process ability. They are recyclable and customized laminates and matrices are available within a wide range. The most significant advantage over thermoset based composites is their weldability [1-5].

A today's barrier for a comprehensive dissemination of TPC structures is the lack of economic, quick and reliable component manufacture processes. To overcome this deficit, fully-automated process chains for the manufacturing and assembly of thermoplastic composites have to be developed in order to achieve production rates and cycle times $(<1 \mathrm{~min})$ required by automotive and aircraft industry as well as the civil engineering sector $[6,7]$. Integrated process cells can be adapted to specific material requirements and to new process elements including laser based techniques for cutting applications [8-10].

Today, different cutting techniques like milling or water jet cutting are used, revealing respective advantages and disadvantages [11-14]. Laser cutting using high power laser sources offers many advantages, such as no tool wear, no water or moisture uptake and highest feed rates. However, the high temperatures reached within the cutting zone could damage the structural properties of the material.

In [15-17] the authors describe the interaction between the laser radiation at different wavelengths and several polymeric materials, comprising unreinforced plastics as well as short and long fibre reinforced composites. As reinforcements, carbon, aramid and glass fibres have been used. In these publications, the focus is mainly put on the investigation of the influence of the applied laser parameters on the resulting cut quality of the respective polymeric material. Therefore, e. g. kerf geometry, roughness, dimension of the burr and global extent of the heat influenced zone have been analyzed.

The aim of this paper is to analyze the influence of the laser impact on TPC laminates with respect to the cohesion from matrix to fibre inside the heat affected zone. In this context, interlaminar shear strength tests are performed. The results are compared to those of conventionally treated samples.

\section{Experimental set-up}

\subsection{Material characterization}

The endless carbon fibre reinforced plastic used within the frame of the investigations is a semicrystalline polyphenylene sulfide (PPS) thermoplastic composite based on high tenacity (HT) fibers. The laminates (consolidated Cetex ${ }^{\circledR}$ plates, provided by TenCate Advanced Composites bv, The Netherlands) consist of harness satin carbon weaves in $0^{\circ} / 90^{\circ}$ fiber orientations $[0 / 90]_{\mathrm{n}}$. The fiber volume content is $V_{\mathrm{G}}=50 \%$ with a mass per unit area of $Q_{\mathrm{C}}=285 \mathrm{~g} / \mathrm{m}^{2}$. The material offers outstanding toughness and excellent chemical and solvent resistance. It is inherently flame resistant with low smoke emission. The cutting experiments presented here were performed using four different laminate thicknesses which are summarized in Table 1. In Figure 1, the fabric structure of the TPC material as schematic drawing as well as a real surface in conjunction with cross sections is shown.

Table 1. Classification of TPC material

\begin{tabular}{|l|c|c|c|c|c|c|}
\hline Laminate thickness & $s$ & {$[\mathrm{~mm}]$} & 0.93 & 1.24 & 2.17 & 3.10 \\
\hline Number of layer & $n$ & {$[-]$} & 3 & 4 & 7 & 10 \\
\hline
\end{tabular}
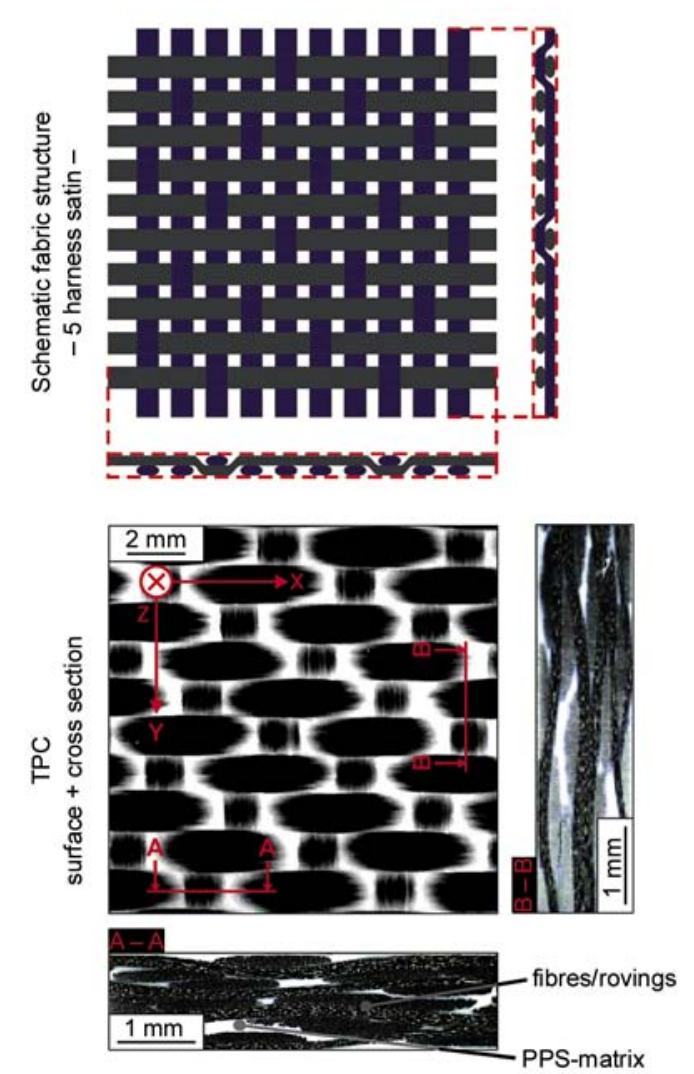

Figure 1. Fabric structure, surface and cross sections of thermoplastic composite laminate in $0^{\circ} / 90^{\circ}$ plate lay-up 


\subsection{Laser source and cutting configuration}

The cutting experiments were performed using a disc laser (HLD 3001.5, Trumpf Laser GmbH + Co. $\mathrm{KG}$, Schramberg, Germany) emitting at a wavelength of $\lambda=1030 \mathrm{~nm}$. The laser radiation is guided by an optical fibre with a diameter of $d_{\text {opt }}=150 \mu \mathrm{m}$ to a laser working head, which focuses the laser beam inside the work piece. The adapted cutting head is based on main components provided by Rofin-Sinar Laser GmbH, Hamburg, Germany. Using a focusing lens with a focal length of $f=$ $200 \mathrm{~mm}$, a laser beam focus of $d_{\mathrm{f}}=150 \mu \mathrm{m}$ is generated. The laser provides an output power of $P_{\mathrm{L}}=$ $2 \mathrm{~kW}$. As process gas, $\mathrm{N}_{2}$ at a working pressure of $p_{\mathrm{N}_{2}}=0.9 \mathrm{MPa}$ was applied. The cutting process was realized by moving the TPC specimen relative to the laser working head using a linear axis (PS01$37 \times 240$, NTI AG - LinMot \& MagSpring, Spreitenbach, Switzerland).

The choice of the applied laser parameter settings will certainly affect the cut quality achieved, and therefore will also affect the static strength of the TPC specimens. To exclude the influence of imperfect cutting parameters and to achieve comparable results to other cutting technologies, optimum parameters were found by parameter variation and then applying them to manufacture the test specimens. The cut quality was rated according to the following criteria: constant laser power independent on the laminate thickness, small kerf width and high optical quality (e.g., little charring). For each material thickness, the maximum feed rate allowing a reliable cutting result was determined, resulting in a specific energy per unit length $E_{\mathrm{s}}$. The relation between $E_{\mathrm{s}}$, the laser power $P_{\mathrm{L}}$ and the feed rate $v_{\mathrm{f}}$ is given in Equation (1):

$$
E_{\mathrm{s}}=\frac{P_{\mathrm{L}}}{v_{\mathrm{f}}}
$$

After identification of the appropriate processing parameters the following dependency between the energy per unit length $E_{\mathrm{s}}$ as well as the number of layers $n$ and the laminate thickness s, respectively, was found (Figure 2).

In Figure 2, a linear dependency between $E_{\mathrm{s}}$ and $n$ becomes apparent. Therefore, an adapted increase of the applied energy in accordance with the laminate thickness is guaranteed.

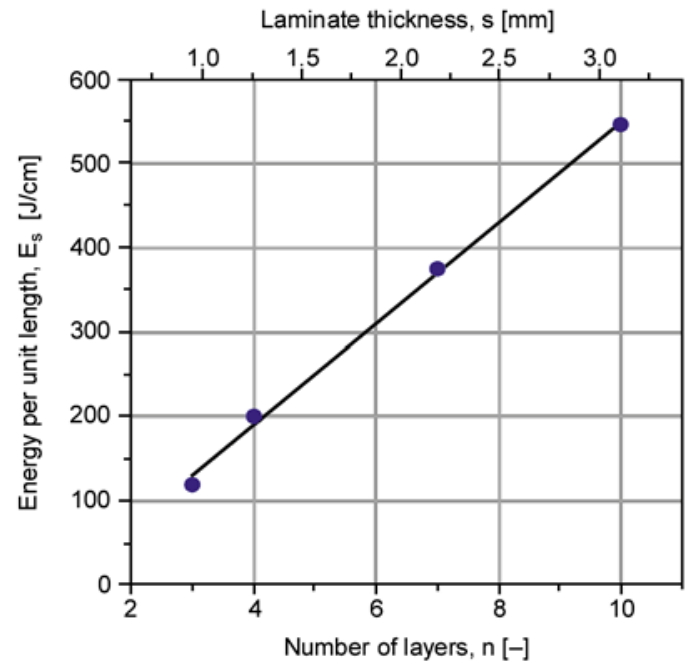

Figure 2. Applied energy per unit length of laser cutting experiments for different TPC laminate thicknesses

\subsection{Material testing}

Interlaminar shear strength tests according to DIN EN 2563 were performed. For reasons of comparison, reference specimens were prepared by milling as well as by abrasive water jet cutting. The milled specimens were manufactured using an FP4 Dialog 11 by DMG, Muenchen, Germany. The water jet equipment consists of a waterknife pump by Flow Europe $\mathrm{GmbH}$, Bretten, Germany in conjunction with a cutting head of BHDT GmbH, Kapfenberg, Austria. Within the frame of the cutting process, garnet sand of Mesh 80 by GMA GARNET (Europe) GmbH, Hamburg, Germany was used.

The specified test method evaluates the quality of the fiber-matrix-adhesion. Therewith, the maximum shear stress of each individual specimen at the moment of the first failure is calculated. First failure occurs if the specimen fails in shear approximately in the neutral axis.

For each thickness of the TPC laminate, specimens at a length of $l_{0}=20 \mathrm{~mm}$ and a width of $b_{0}=10 \mathrm{~mm}$ were prepared. The length of the specimen is parallel to the warp direction of the fabrics. The rectangular specimen is positioned perpendicular and tested of flexure on two supports with a radius of $r_{\mathrm{t}}=$ $3 \mathrm{~mm}$ and a distance of $l_{\mathrm{t}}=10 \mathrm{~mm}$ between them. The load is applied at the center of the specimen by means of a loading nose with a same radius $r$ at a constant displacement rate of $v_{\mathrm{t}}=1 \mathrm{~mm} / \mathrm{min}$ until the moment of first failure. The corresponding bending strain is measured simultaneously. 
The bending tests were performed using a universal test machine Zwick Z250 providing an accuracy of $1 \%$ of the load range in conjunction with a control software Test XPert 11.0, which records the load applied as a function of displacement of the loading nose. The tested specimens were inspected by preparation of optical micrographs in order to make sure that only interlaminar failure occurred, so fibre breakage has no influence on the bending results. In this case the interlaminar shear strength can be calculated according to DIN EN 2563.

Each test result was verified by testing seven identical specimens. All tests were performed at standard atmosphere with $23^{\circ} \mathrm{C}$ and $50 \%$ humidity. In order to visualize the cutting zone, optical micrographs were prepared.

\section{Results and discussion}

In the following, the quality of the laser cuts achieved is discussed and compared to the cut quality of conventional machining with a special emphasis on the extent of the thermal damage to the TPC. The results from the static interlaminar shear tests are given and correlated with the cut qualities identified.

\subsection{Achievable cut quality}

As expected, milling as well as abrasive water jet cutting produces cuts of high optical quality. No delamination or free fibre ends were observed. The laser cut samples clearly show an extended heat affected zone starting from the cutting kerf into the

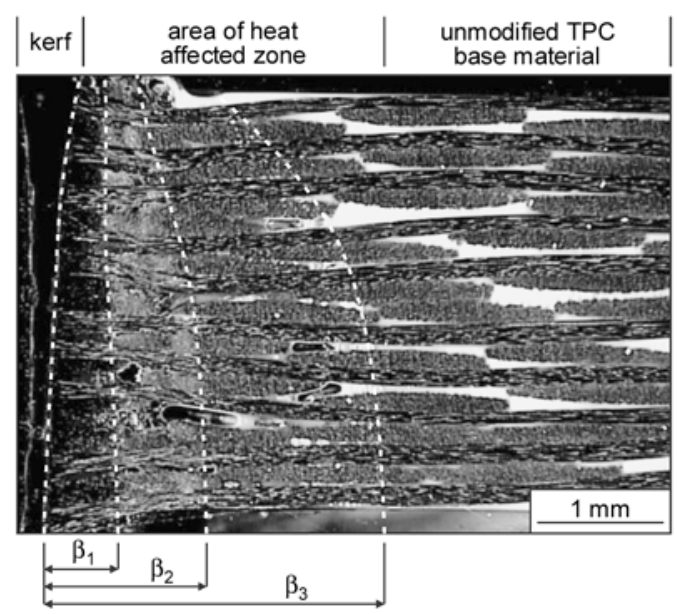

Figure 3. Optical micrograph (cross section) of laser cut TPC specimen for an $n=10$ layer arrangement and characteristic regions within the heat affected zone identified thermoplastic composite with changed material properties, visible by varying light reflections. In Figure 3, a cross section of a TPC disc laser cut generated using a laser output power of $P_{\mathrm{L}}=2 \mathrm{~kW}$ for an $n=10$ layer arrangement with a thickness of $s=3.1 \mathrm{~mm}$ is shown.

Different areas within the HAZ have been identified, in which the material damage occurs in a different manner. The induced temperature gradient, arising from the high thermal conductivity of the HT-fibres along the fibre axis $\left(\lambda_{\mathrm{c}}=17 \mathrm{~W} /(\mathrm{m} \cdot \mathrm{K})\right)$ and starting at the cutting kerf towards the inner bulk material, influences the composition of the thermoplastic matrix system. Close to the cutting edge, damaged areas are detected between the single carbon fibre fabrics (interlaminar) as well as within the rovings themselves. In this zone, the PPS matrix is vaporized and the carbon fibres are charred $\left(\beta_{1}\right)$. Adjacent to this area, it is supposed that mainly matrix damage occurs due to exceeding the decomposition temperature of $T_{\mathrm{V}}=370^{\circ} \mathrm{C}$ for PPS $\left(\beta_{2}\right)$. An in-situ determination of the process temperature within the composite has not been realized. With increasing distance to the cutting edge, pure interlaminar effects, i.e. structural modification within the PPS matrix depots between the carbon fibre rovings, become visible. Porosities are detected within the polymer matrix, whereas the carbon fibre fabric remains intact $\left(\beta_{3}\right)$. The inclusion of pores within TPC laminate structures is well known from the thermoforming process of such materials, in case of applying insufficient molding pressures during the consolidation phase. In this context, the pores are generated due to mechanical inclusion of air after reaching the PPS melting temperature of $T_{\mathrm{m}}=285^{\circ} \mathrm{C}$. Therefore, it can be stated that the intersection between HAZ and unmodified bulk material is determined by re-solidification of the polymeric PPS matrix.

A quantitative analysis of the laser cut specimens revealed an increase of the HAZ with increasing material thickness at constant $P_{\mathrm{L}}$. In Figure 4, the extensions of the different areas identified within the HAZ are presented for all 4 layer arrangements. For the evaluation of the respective HAZ area $\beta_{\mathrm{k}}$, $k \in\{1,2,3\}$, the micrographs were separated into 3 horizontal sectors, which were measured independently of each other, providing a mean value for each material thickness. 


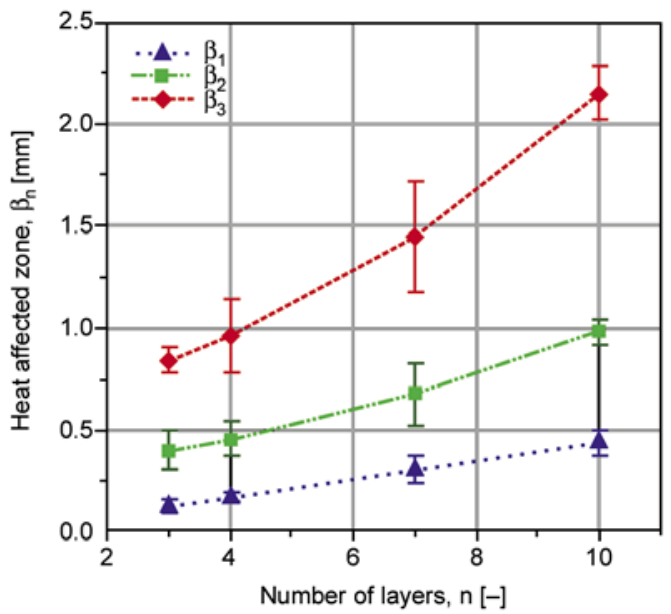

Figure 4. Different regions of the heat affected zone as a function of material thickness

\subsection{Interlaminar shear strength tests}

In order to investigate the influence of the resulting HAZ during laser cutting of TPC on the material properties, interlaminar shear stress measurements were performed. For all composite thicknesses, seven specimens were tested. As reference for the laser cut samples, additional specimens were prepared by milling as well as abrasive water jet cutting technique. The corresponding results of the interlaminar shear strength investigations are shown in Figure 5. The mean values and the respective standard deviations are indicated. For a better overview, within Figure 5 as well as within all following figures, the determined measuring values are spread around the respective center number of layer. All measurements were performed for $n=3$, 4, 7 and 10 layers.

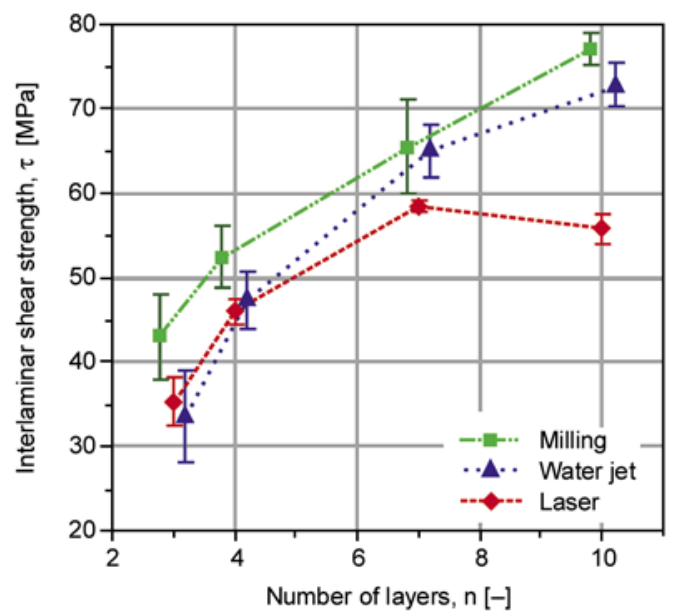

Figure 5. Interlaminar shear strength for different processing technologies as a function of laminate thickness
Overall, an increase of the interlaminar shear strength $\tau$ with increasing thickness of the TPC laminate becomes obvious. Since $\tau$ depends on the size of the load bearing area which is determined by the width and the thickness of a respective specimen, this measuring value is no material constant but mainly used for comparative investigations. In this case $\tau$ is used for a comparison of laser and conventionally treated specimens. The highest interlaminar shear strengths $\tau$ are observed for milled specimens. For laminate arrangements consisting of 3 and 4 layers, abrasive water jet and laser machined specimens reveal interlaminar shear strengths on a similar level. With increasing material thickness, the measured interlaminar shear strengths of laser cut specimens decreases in terms of the curve progression of the water jet treated samples.

In Figure 6, the corresponding bending strains $\varepsilon$ for tested specimens generated with all 3 cutting techniques are shown. The values of milled as well as abrasive water jet machined specimens reveal a comparatively constant behaviour, independent on the material thickness. In contrast, for laser cut CF PPS, the bending strain significantly increases with increasing number of layers. A linear relation between $\varepsilon$ and $n$ becomes apparent. Overall, the laser processed specimens show higher bending elongations compared to conventionally treated samples.

In order to analyze the reduction of the interlaminar shear strength in case of laser treatment, the differences in $\tau$ with respect to milling and abrasive water jet machining are calculated. In Figure 7, these differences $\Delta \tau$ are plotted against the number of layers

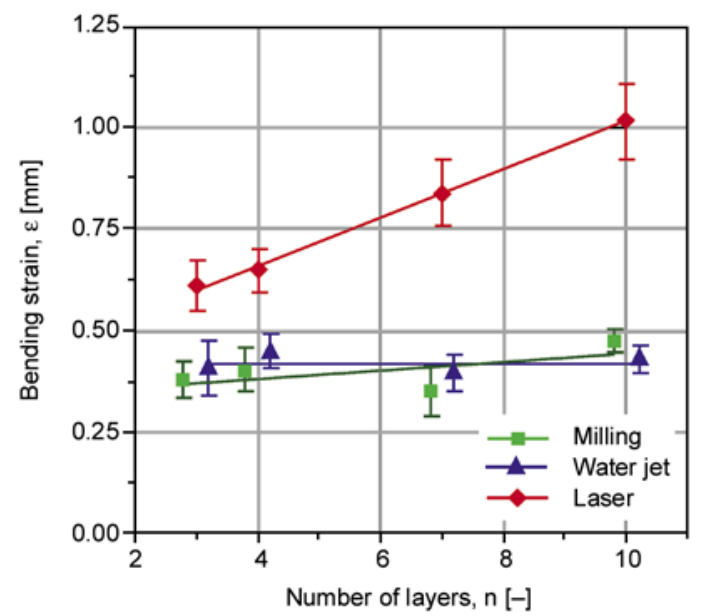

Figure 6. Bending strain for different processing technologies as a function of laminate thickness 


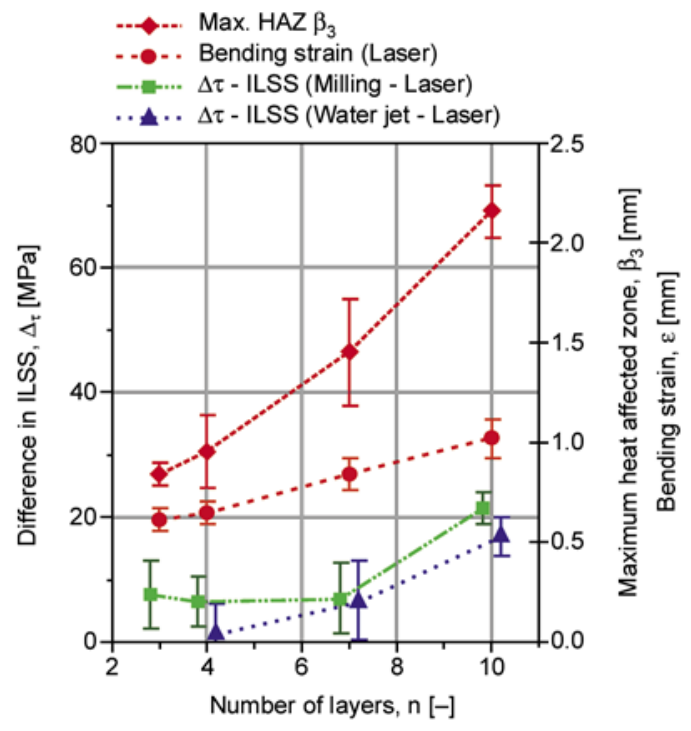

Figure 7. Bending strain and maximum extent of HAZ of laser processed specimens as well as differences in interlaminar shear strength between laser and conventionally treated CF PPS as a function of laminate thickness

$n$. Additionally, the bending strain $\varepsilon$ as well as the maximum extent of the heat affected zone $\beta_{3}$ are added into the graph. Two main aspects can be drawn from the data. Firstly, with increasing extent of the heat affected zone, the measured bending strain increases as well. Secondly, the progression of the bending elongation of laser processed TPC specimens is directly mirrored in the difference curves of the interlaminar shear strengths $\Delta \tau$. Obviously, the larger the influence of the thermal cutting process on the material properties of the composite laminate, the lower the interlaminar shear strength and the higher the measured corresponding bending strain.

Taking into account the resulting heat affected zone due to laser impact, it is assumed that the HAZ partially reduces the load bearing area of the material as a consequence of missing or reduced fibrematrix-adhesion at the edges of the specimens. As a result of this, the effective width of the laser cut specimens within the bending test is smaller compared to those of the conventionally machined samples. Correspondingly, the bending strain $\varepsilon$ increases in case of laser cut TPC.

For bending tests, the interlaminar shear strength $\tau_{0}$ can be derived from Equation (2), where $F_{\max }$ is the maximum bending force applied at the respective failure of the sample, $s$ the thickness and $b_{0}$ the width of the test specimen. $\tau_{0}=\frac{3 \cdot F_{\max }}{4 \cdot s \cdot b_{0}}$

Considering the formation of a heat influenced zone at the edges of the specimen in case of laser treatment, Equation(2) can be expressed as Equation (3):

$\tau_{\text {eff }}=\frac{3 \cdot F_{\max }}{4 \cdot s \cdot b_{\text {eff }}}$

comprising an actual interlaminar shear strength $\tau_{\text {eff }}$ and an adapted specimen width $b_{\text {eff, }}$ which is a function of the extent of the HAZ. Therefore, $b_{\text {eff }}$ can be formulated as Equation (4):

$b_{\text {eff }}=b_{0}-2 \cdot \beta_{\mathrm{k}}$ with $k \in\{1,2,3\}$

Using Equation (3) and (4), actual interlaminar shear strengths were calculated in consideration of $\beta_{\mathrm{k}}$, which were derived from cross sections analogue to the approach presented in Figure 3 and for which the corresponding mean values are given in Figure 4. In Figure 8, the resulting curve progressions for $\tau_{0}$ as well as for $\tau_{\text {eff }}$ for the areas $\beta_{1}$ and $\beta_{2}$ of the HAZ at varying laminate thickness are shown. In accordance with the prediction with respect to a reduced load bearing area as a consequence of laser impact, a significant increase of $\tau_{\text {eff }}$ especially for higher $n$, corresponding to a higher degree of material damage becomes apparent. In relation to the interlaminar shear strengths achieved for conventionally processed specimens (Figure 5), it can be stated that $\tau_{\text {eff }}\left(\beta_{2}\right)$ is found on a same level. Solely, for the $n=10$ layer laminate, $\tau_{\text {eff }}\left(\beta_{2}\right)$ still declines

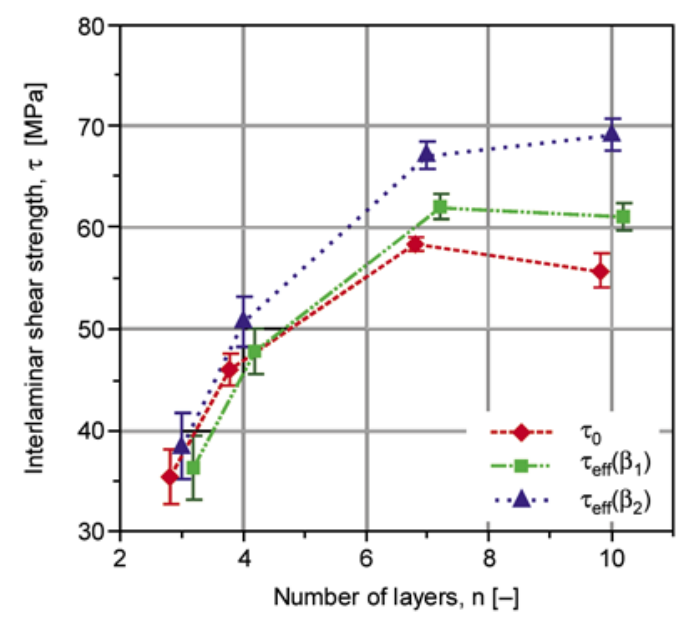

Figure 8. Progression of actual interlaminar shear strength for laser machined specimens as a function of laminate thickness 
to values of approx. $\tau_{\text {eff }}=70 \mathrm{MPa}$, which is still lower than milled or water jet treated samples.

Since $\tau_{\text {eff }}\left(\beta_{2}\right)$ already reaches the range of achievable interlaminar shear strengths provided by conventional cutting processes, $\beta_{2}$ of the $\mathrm{HAZ}$ as crucial value determining the degree of decreased load bearing area is deduced. It can be stated that the area of re-solidified polymeric PPS matrix of the HAZ $\left(\Delta \beta_{32}=\beta_{3}-\beta_{2}\right)$ marginally influences the load transmission during the bending test. This is applicable for thin laminates, $n \leq 7$ layers, whereas for thicker composite structures, a trend towards a deeper interlaminar damage due to laser impact in conjunction with extensive heat conduction is found. However, even for $n=10, \tau_{\mathrm{eff}}\left(\beta_{3}\right)$ provides interlaminar shear strengths far above the curves of milling as well as abrasive water jet processed samples, for which reason the corresponding data for $\beta_{3}$ is excluded in Figure 8. Overall, the use of high power laser cutting technique is of special interest in case of thin composite laminates, for which this technique is most applicable.

\section{Conslusions}

Within this paper, investigations on whether thermal damage by high power laser processing reduces the interlaminar shear strength properties of endless carbon fibre reinforced thermoplastic composite materials are given. To achieve this, test specimens were cut from semicrystalline polyphenylene sulfide composite laminates based on HT fibres in harness satin carbon fabrics in $[0 / 90]_{\mathrm{n}}$ fiber orientations with a thickness of 0.93 to $3.1 \mathrm{~mm}$. The following conclusions can be drawn from the investigations:

1. For laser machined specimens, a heat affected zone (HAZ) starting at the cutting edge can clearly be detected. A quantitative analysis of the laser cut specimens revealed an increase of the HAZ with increasing material thickness at constant laser output power. The HAZ is classified into three areas, in which the material damage occurs in a different manner.

2. Interlaminar shear strength tests reveal a reduction of the maximum shear stresses for laser cut specimens compared to conventionally treated samples, provided by milling as well as abrasive water jet technique. Within the laser working range, an increase of the achievable shear strengths for a distinct material thickness with decreasing extent of the HAZ is found. Therefore, for comparably thin laminate arrangements, interlaminar shear strengths of laser machined specimens converge to those of conventionally treated TPC samples.

3. The corresponding bending strains reveal a comparatively constant behaviour for conventionally processed specimens, independent on the material thickness. In contrast, for laser cut CF PPS, the bending strain significantly increases with increasing material thickness and caused lower $E$-modulus. For specified loading conditions, e.g. about impacts, the laser processed specimens show higher bending elongations compared to conventionally treated samples.

4. Taking into account the different areas of the HAZ, actual interlaminar shear strengths for laser treated specimens have been calculated. A reduced load bearing area has been identified as main factor for the reduction of interlaminar shear strengths achieved.

\section{Acknowledgements}

The authors would like to thank the German Research Foundation (DFG) for their support within the project HA $1213 / 74$ 1. Furthermore, the authors would like to thank Dr. A. Schenk of the Water Jet Laboratory Hanover for kindly machining the reference specimens by abrasive water jet cutting. Finally the authors would like to express their gratitude to TenCate Advanced Composites bv, The Netherlands, for the supply of the consolidated Cetex ${ }^{\circledR}$ PPS composite laminates.

\section{References}

[1] Jaeschke P., Herzog D., Kern M., Erciyas A. S., Peters C., Purol H., Herrmann A. S.: Laser transmission welding of thermoplastic composites - Fundamental investigations into the influence of the carbon fibre reinforcement and orientation on the weld formation. Joining Plastics, 4, 247-255 (2009).

[2] Jaeschke P., Herzog D., Hustedt M.: Thermography aids development of laser transmission welding. Plastics Engineering, 7, 28-34 (2009).

[3] Offringa A.: Thermoplastics in aerospace, a stepping stone approach. in 'Proceedings of $1^{\text {st }}$ Cetex Conference. Delft, Netherlands' 1-13 (2006).

[4] Kelly G.: Joining of carbon fibre reinforced plastics for automotive applications. PhD Thesis, Royal Institute of Technology, Stockholm, Sweden (2004). 
[5] van Wijngaarden M. J.: Robotic induction welding of carbon fiber reinforced thermoplastics. In 'Proceedings of $1^{\text {st }}$ Cetex Conference. Delft, The Netherlands' 149-160 (2006).

[6] Purol H., Peters C., Herrmann A. S.: Fast manufacturing of thermoplastic parts and challenges for a fullyautomated process chain. in 'International Conference on Applied Production Technology. Bremen, Germany' 53-58 (2007).

[7] Peters C., Purol H., Herrmann A. S.: Integrated thermoforming process for CFRP-parts mass production. in 'Japan International SAMPE Technical Seminar. Kyoto, Japan’ Paper No. 8 (2008).

[8] Herzog D., Jaeschke P., Meier O., Haferkamp H.: Investigations on the thermal effect caused by laser cutting with respect to static strength of CFRP. International Journal of Machine Tools and Manufacture, 48, 1464-1473 (2008).

DOI: 10.1016/j.ijmachtools.2008.04.007

[9] Jaeschke P., Herzog D., Noelke C., Henning B., Haferkamp H.: Investigations into the sealing of heat damaged areas by applying polymer powders during laser cutting of carbon fiber reinforced composites. Advanced Engineering Materials, 12, 587-590 (2009). DOI: 10.1002/adem.200900310

[10] Jaeschke P., Hustedt M., Herzog D.: Ablation of cured prepreg laminates using laser pulsed water jets. in 'Proceedings of $15^{\text {th }}$ International Conference on Composite Structures. Porto, Portugal' Paper No. 344 (2009).
[11] Koplev A., Lystrup A., Vorm T.: The cutting process, chips, and cutting forces in machining CFRP. Composites, 14, 371-376 (1983).

DOI: $10.1016 / 0010-4361(83) 90157-X$

[12] Hu N. S., Zhang L. C.: Some observations in grinding unidirectional carbon fibre-reinforced plastics. Journal of Materials Processing Technology, 152, 333-338 (2004).

DOI: $10.1016 /$ j.jmatprotec.2004.04.374

[13] Davim J. P., Reis P.: Damage and dimensional precision on milling carbon fiber-reinforced plastics using design experiments. Journal of Materials Processing Technology, 160, 160-167 (2005).

DOI: 10.1016/j.jmatprotec.2004.06.003

[14] Moeller R.: Machining of composites with abrasive waterjets. in 'Proceedings of CFK-Valley Stade Convention. Stade, Germany’ 48-49 (2007).

[15] Davim J. P., Barricas N., Conceicao M., Oliveira C.: Some experimental studies on $\mathrm{CO}_{2}$ laser cutting quality of polymeric materials. Journal of Materials Processing Technology, 198, 99-104 (2008).

DOI: 10.1016/j.jmatprotec.2007.06.056

[16] Mathew J., Goswami G. L., Ramakrishnan N., Naik N. K.: Parametric studies on pulsed Nd:YAG laser cutting of carbon fibre reinforced plastic composites. Journal of Materials Processing Technology, 89, 198-203 (1999).

DOI: 10.1016/S0924-0136(99)00011-4

[17] Goeke A., Emmelmann C.: Influence of laser cutting parameters on CFRP part quality. Physics Procedia, 5, 253-258 (2010).

DOI: $10.1016 / \mathrm{j}$. phpro.2010.08.051 